\title{
Response to: Arthropathy-like findings and a carpal tunnel syndrome as the presenting features of Scheie syndrome: Three cases from the same family
}

\author{
Domingo Ly-Pen ${ }^{1}$, Jose-Luis Andreu ${ }^{2}$ \\ ${ }^{1}$ Abbey House Medical Centre, Abbey Road, Navan. Co Meath. Navan, Ireland; ${ }^{2}$ Department of Rheumatology, \\ Hospital Universitario Puerta de Hierro Majadahonda, C/ Manuel de Falla 1; 28222, Majadahonda. Madrid, Spain. \\ E-mail: domingoly@gmail.com \\ Received: 2nd April 2019, Accepted: 3rd April 2019
}

\section{Dear Editor,}

We have read with great interest the recent article "Arthropathy-like findings and a carpal tunnel syndrome as the presenting features of Scheie syndrome: Three cases from the same family", by Gökay et al. ${ }^{1}$ We are pleased that they published with regard to this unusual cause of carpal tunnel syndrome (CTS), seen more often in children with other pathologies. We are sure that if clinicians bear in mind the possibility of Scheie syndrome in CTS in children and young patients, more cases would be diagnosed, and early therapy could be initiated, with better prognosis.

We would like to bring to attention our study, not yet fully published, but that was presented in an EULAR (EUropean League Against Rheumatism) meeting. ${ }^{2}$ This was a protocol for early diagnosis of the atenuated form of Mucopolysaccharidosis I (MPS I) or Scheie syndrome in young patients (less than 30 years old) diagnosed with CTS.

We lack accurate data that could assess the epidemiology of Scheie syndrome, especially in Spain (where our study was conducted). Prognosis of the disease greatly depends upon early diagnosis. These facts and the availability of a rapid, sensitive and reliable test on Dry Blood Spot Filter Paper (DBSFP) for identifying $\alpha$-L-Iduronidase deficiency ${ }^{1}$ and the existence of an Enzymatic Replacement Treatment for the disease make an early diagnosis of these patients dramatically important. CTS is among one of the most well-known and common findings in MPS $\mathrm{I}^{3}$ Of the "natural history" patients who reported on carpal tunnel status, $33 / 110(30 \%)$ of Hurler patients, 60/86 (70\%) of Hurler-Scheie patients, and 54/60 (90\%) of Scheie patients, reported having $\mathrm{CTS}^{3}$; nonetheless CTS is very uncommon in infancy and young adulthood. ${ }^{3}$

We conducted a cross-sectional prospective study of a cohort of patients with an electromyogram-confirmed diagnosis of CTS. Our total population is over 600.000 people of an urban area of Madrid. We included patients in two ways: 1) searching in the "Ramón y Cajal" Hospital database for patients diagnosed with CTS under the age of 30 years, 2) searching for new patients with suggestive symptoms: Children and young adults with joint stiffness, flexion contractures, claw hand, trigger fingers, thenar atrophy, poor hand function, hand paresthesia. Both groups of patients were offered the DBSFP test to confirm Scheie syndrome. Chi square for categoric variables and Student $\mathrm{T}$ analysis or ANOVA test for quantitative variables were used. The study was approved by the ethics committees at our centers and all patients (or legal guardians) gave written informed consent before inclusion in study.

Twelve patients (range: 8-28 years old, media and median: 23 years) were included. Two patients had low $\alpha$-L-Iduronidase activity (5\% and $40 \%$ ) in the DBSFP test. The results of enzymatic activity of $\alpha$-L-Iduronidase in lymphocytes, were normal.

Despite the fact that we were not able to diagnose new cases of Scheie syndrome, because our sample was too small, we believe and fully agree with Gökay et al, ${ }^{1}$ that: "Scheie syndrome may be challenging because patient's appearance can be completely normal, and CTS 
may be the only clue of diagnosis. Recognition of key symptoms and early diagnosis is the only way to prevent possible complications for this rare but treatable disease."

\section{REFERENCES}

1. Gökay S, Kardaş F, Kendirci M, Sözeri B. Arthropathylike findings and a carpal tunnel syndrome as the presenting features of Scheie syndrome: Three cases from the same family. Turk J Pediatr 2018; 60: 344347.
2. Ly-Pen D, Andreu J, de Blas G, Sanchez-Olaso A, Jiménez San-Emeterio J. Prevalence of Mucopolysaccharidosis I in a pediatric and young adult population diagnosed with carpal tunnel syndrome [abstract] Ann Rheum Dis 2010; 69(Suppl 3): 113.

3. Van Meir N, De Smet L. Carpal tunnel syndrome in children. Acta Orthop Belg 2003; 69: 387-395. 